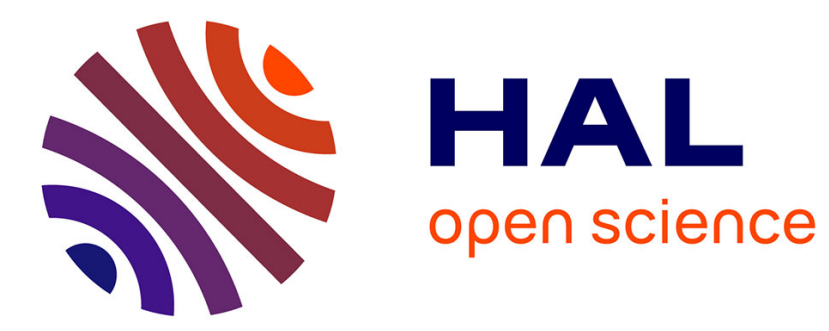

\title{
New absorbing layers conditions for short water waves
}

Hélène Barucq, Julien Diaz, Mounir Tlemcani

\section{To cite this version:}

Hélène Barucq, Julien Diaz, Mounir Tlemcani. New absorbing layers conditions for short water waves. Journal of Computational Physics, 2010, 229, pp.58-72. 10.1016/j.jcp.2009.08.033 . inria-00418317

\section{HAL Id: inria-00418317 https://hal.inria.fr/inria-00418317}

Submitted on 18 Sep 2009

HAL is a multi-disciplinary open access archive for the deposit and dissemination of scientific research documents, whether they are published or not. The documents may come from teaching and research institutions in France or abroad, or from public or private research centers.
L'archive ouverte pluridisciplinaire HAL, est destinée au dépôt et à la diffusion de documents scientifiques de niveau recherche, publiés ou non, émanant des établissements d'enseignement et de recherche français ou étrangers, des laboratoires publics ou privés. 


\title{
New absorbing layers conditions for short water waves
}

\author{
H. Barucq ${ }^{\mathrm{a}, \mathrm{b}}$, J.Diaz $^{\mathrm{a}, \mathrm{b}}$, M. Tlemcanic \\ ${ }^{a}$ INRIA Bordeaux Sud-Ouest Research Center Team-Project Magique3D \\ ${ }^{b}$ Laboratoire de Mathématiques Appliquées, CNRS UMR 5142, Université de Pau et des \\ Pays de l'Adour, IPRA-Avenue de l'Université, 64013 Pau, France \\ ${ }^{c}$ Département de Physique, Faculté des Sciences,BP 1505 El M'Naouer (USTOMB), \\ ORAN 1300, Algérie
}

\begin{abstract}
We develop a new PML formulation for the linearized shallow-water equations including the Coriolis force. The construction process is based on the uncoupling of the velocity components with the depth of water. Then the damping effect is only applied to the propagative modes just as was formerly done by Nataf [1] to the linearized Euler equations to enforce the long-time stability. We assess numerically the performance of the new absorbing condition and we illustrate in particular that it is stable for long-time simulations.
\end{abstract}

Key words: Linearized Shallow Water Equations, Coriolis Force, Perfectly Matched Layer.

\section{Introduction}

Many applications require to solve numerically dispersive wave problems in a domain which is much smaller than the physical one. A current method consists in applying a local absorbing boundary condition (ABC) on the exterior boundary which is used to limit the computational domain. The ABC should minimize spurious reflections when waves impinge on the exterior boundary which has no physical meaning. Obviously, the more efficient the ABC is, the more accurate the numerical solution will be. A lot of works have been dealing with the construction of $\mathrm{ABCs}$ that after discretization lead to a stable and accurate scheme and we refer to $[2,3]$ for discussion on related issues and for reviews on the subject. Most of the ABCs have been designed for either timeharmonic waves or for nondispersive time-dependent waves and the use of ABCs in case of dispersive waves is much more difficult. A important example where dispersive waves must be considered is that of meteorological models which take into account the Earth's rotation [4]. Recently, Van Joolen et al. [5] have developed a new numerical scheme including high-order $\mathrm{ABCs}$ for dispersive waves

Email address: helene.barucq@inria.fr, julien.diaz@inria.fr, mounir.tlemcani@univ-pau.fr (M. Tlemcani)

Preprint submitted to computational physics

July 16, 2009 
which are based on Higdon's ABCs [6] . While it is possible to construct local ABCs easy to implement, their efficiency to damp spurious reflections often suffer from the corner problem. This difficulty can be overcome by using Perfectly Matched Layers just as was suggested by Bérenger [7, 8]. The very attractive property of the PML is it absorbs all the waves without spurious reflection and the corner problem is easily solved by a suitable fit of the layer parameters. Moreover, the coupling of the physical system with the PML condition is easy to handle numerically. Many works have been devoted to the design of PML for various applications and as far as the Shallow Water equations are concerned, Navon et al. [9] have recently developed a split Perfectly Matched Layer (PML) scheme for the linearized system which is based on an explicit finite-difference discretization scheme. This PML requires a stabilization process involving a 9point Laplacian filter to avoid the split PML supports unstable solutions. This question was formerly adressed for the linearized Euler equations in [10] and next in [11] for more general flows. Both in [10] and [11], the PML is obtained via a change of coordinate in the complex plane applied to the direction normal to the boundary. This amounts to replacing all the normal-derivatives in the Euler system by an operator which is still differential in the normal-direction but pseudo-differential in the other variables. In [1], F. Nataf has proposed another strategy which leads to the design of a stable PML for the Euler equation. The idea consists in applying the Smith factorization to the Euler equations in order to uncouple the propagative part of the solution from the transport one. Then the PML is constructed in such a way that only the modes that could produce reflections are damped. Thus the vorticity modes, which satisfy transparent conditions [12] on the computational boundary domain, are not damped and the resulting scheme seems to be stable as numerical experiments show.

In this paper, we propose a new PML formulation of the linearized shallowwater equations whose construction is based on the splitting of the primal system. Under the assumption the Coriolis force is constant, the depth $h$ can be uncoupled from the velocity components by applying some elementary combinations which preserve the differential structure of the initial equations and the resulting formulation involves now a Klein-Gordon equation. The decomposition results in uncoupling the advective part of the wave from the vorticity (section 2). After having remarked that the vorticity waves can be absorbed via an appropriate transparent condition and therefore do not need to be damped in the artificial layer, the PML condition is written by applying a complex coordinate change to the advective unknown only (section 3 ). In section 4 we discuss the practical handling of the method and we present the numerical scheme used to discretize the PML equations. Numerical results are presented in section 5. They confirm the new layer is perfectly matched to the physical domain and by considering long times of simulation, they seem to illustrate the long-time stability of the PML system. 


\section{Setting of the primal system}

The shallow-water model contains some of the important dynamical features of the atmosphere and ocean and experience has shown that it is capable of describing main aspects of their motions. Let us consider a fluid with constant and uniform density. The height of the fluid surface above the reference level $z=0$ is $h:=h(t, x, y)$. Even if $h$ varies in space and time, we suppose that we can choose a characteristic value for the depth which is denoted by $H$. In the same way, we assume there exists a characteristic horizontal length scale for the motion which we call $L$. Then the fundamental condition which characterizes shallow-water theory is

$$
\delta=\frac{H}{L}<<1
$$

We suppose the rotation axis of the fluid coincides with the $z$-axis so that the Coriolis parameter $f$ is a constant. The velocity has components $u, v$ and $w$ parallel to the $x$-, $y$ - and $z$-axis respectively. The pressure of the fluid can be arbitrarily imposed and herein, we take it to be constant which implies in particular that the horizontal pressure gradient is independant of $z$. It is therefore consistent to assume that the horizontal velocities themselves are $z$ independent if they are at $z=0$. Moreover, $w$ can be uncoupled from $u$ and $v$, observing that this simplification is no more possible if the density varies with $z$. At last, the fluid is supposed to be inviscid, which amounts to neglect viscosity effects. According to [4], the linearized horizontal momentum equations read then as:

$$
\left\{\begin{array}{l}
G h+H\left(\partial_{x} u+\partial_{y} v\right)=0 \\
G u+\partial_{x} h-f v=0 \\
G v+\partial_{y} h+f u=0
\end{array}\right.
$$

where $G$ denotes the transport operator defined by :

$$
G=\partial_{t}+U \partial_{x}+V \partial_{y}
$$

and $U, V$ and $H$ are positive constants which characterize the equilibrium state around which the linearization process has been done. In this paper, we consider the subsonic case corresponding to $U^{2}+V^{2}<H$.

Under the assumption $f$ is constant, the unknown $h$ can be uncoupled from the two other ones by using some combinations of the three equations. Indeed, by deriving the second (resp. third) equation of (2) with respect to $x$ (resp. $y$ ), and by summing up the two resulting equations, we obtain:

$$
G\left(\partial_{x} u+\partial_{y} v\right)=-\Delta h+f\left(\partial_{x} v-\partial_{y} u\right)
$$

Then by applying $G$ to the first equation and by plugging (3) into the resulting equation, we have:

$$
\left(G^{2}-H \Delta\right) h+H f\left(\partial_{x} v-\partial_{y} u\right)=0
$$


Next, by deriving the second (resp. third) equation of (2) with respect to $y$ (resp. $x$ ), we get a writting of $G\left(\partial_{x} v-\partial_{y} u\right)$ which can be used after applying $G$ to (4). We thus get that $h$ is solution to the third-order differential equation:

$$
\left(G^{2}-H \Delta\right)(G h)+f^{2}(G h)=0
$$

which shows that $h^{*}=G h$ is solution to the second-order equation:

$$
\left(G^{2}-H \Delta\right) h^{*}+f^{2} h^{*}=0
$$

The second-order operator $G^{2}-H \Delta+f^{2}$ is currently refered to as the KleinGordon operator and $G^{2}-H \Delta$ will be denoted by $L$ in the following. Now, the shallow-water equation can be rewritten as:

$$
\left\{\begin{array}{l}
L h^{*}+f^{2} h^{*}=0 \\
G u+\partial_{x} h-f v=0 \\
G v+\partial_{y} h+f u=0
\end{array}\right.
$$

with the condition $h^{*}=G h$.

Remark 2.1. The previous system can be obtained by applying a Smith factorization [13] to the shallow-water equation, just as was formerly done in [1] for the linearized Euler equations.

The new formulation is interesting since it allows one to discriminate the two types of fields which define a solution to the shallow water equation. Indeed, the first equation is the only one to describe a propagation phenomenon while the two other ones are transport equations. Hence, only the first one can induce reflections and it implies that the PML condition will be applied to $h^{*}$ only and not the whole solution $\left(h^{*}, h, u, v\right)$.

\section{The new PML model}

Just as was previously mentionned, we focus on the description of a PML acting in the $x$-direction but what follows is not restrictive since it is sufficient to exchange $x$ and $U$ for $y$ and $V$ to obtain the corresponding PML in the $y$-direction.

The construction of the PML model is based on substituting only the $x$ derivatives acting on $h^{*}$ into the first equation of (7) by a PML-derivative. By this way, we aim at enforcing the stability of the PML model which is not guaranteed by substituting the $x$-derivative by the PML-derivative everywhere. Such an approach has been successfully used by Nataf [1] for the linearized Euler

equation and herein, we apply the same derivation process than in [1] where the PML-derivative is defined by:

$$
\partial_{x}^{\mathrm{PML}}=\alpha\left(x, \partial_{t}, \partial_{y}\right)\left(\partial_{x}-\frac{U}{H-U^{2}}\left(\partial_{t}+V \partial_{y}\right)\right)+\frac{U}{H-U^{2}}\left(\partial_{t}+V \partial_{y}\right)
$$


The operator $\alpha$ depends on a damping parameter $\sigma:=\sigma(x) \geq 0$ and is formally defined as follows:

$$
\alpha\left(x, \partial_{t}, \partial_{y}\right)=\frac{\sqrt{H}\left(\partial_{t}+V \partial_{y}\right)}{\sqrt{H}\left(\partial_{t}+V \partial_{y}\right)+\left(H-U^{2}\right) \sigma(x)}
$$

Then, denoting by $L^{\mathrm{PML}}$ the operator $L$ in which $\partial_{x}$ has been replaced by $\partial_{x}^{\mathrm{PML}}$, the coupling of the PML condition with system (7) gives rise to the new system:

$$
\left\{\begin{array}{l}
L^{\mathrm{PML}} h^{*}+f^{2} h^{*}=0 \\
G u+\partial_{x} h-f v=0 \\
G v+\partial_{y} h+f u=0
\end{array}\right.
$$

with the condition $h^{*}=G h$.

Next, we address the question of verifying if the new model is a PML one in the sense that no reflection occurs at the interface between the exact medium and the absorbing one. Hence, we have to consider the coupling of the initial model (7) with the new one (10) when the PML medium is defined for instance by the half-space $x>0$ while the physical one is given by $x<0$ and to check that the transmission accross $\{x=0\}$ is perfect. We have:

Theorem 3.1. The two systems (7) for $x<0$ and (10) for $x>0$ are perfectly matched if they are coupled by the transmission conditions $\left[h^{*}\right]=0,\left[D_{x} h^{*}\right]=0$, $[u]=0$ and $[v]=0$ at the interface $x=0$ between the two media, where $D_{x}$ is defined as $\partial_{x}$ for $x<0$ and $\partial_{x}^{P M L}$ for $x>0$.

Proof 3.2. We have already remarked that the field $h^{*}$ satisfies a Klein-Gordon equation independently of the field $(u, v)$. Moreover, the field $(u, v)$ is solution to a system of coupled transport equations for which the transmission conditions $[u]=0$ and $[v]=0$ at $x=0$ are transparent. Hence it is sufficient to verify that the two first equations of (7) and (10) are perfectly matched if $h^{*}$ satisfies the transmission conditions $\left[h^{*}\right]=0$ and $\left[D_{x} h^{*}\right]=0$ at $x=0$.

We then consider the change of variable

$$
\left\{\begin{array}{l}
t=\frac{\sqrt{H}}{H-U^{2}} t_{1}-\frac{U}{H-U^{2}} x_{1}, \\
x=x_{1}, \\
y=\sqrt{H-U^{2}} y_{1}-\frac{U V}{H-U^{2}} x_{1}+\frac{V \sqrt{H}}{H-U^{2}} t_{1}, \\
h_{1}^{*}\left(x_{1}, y_{1}, t_{1}\right)=h^{*}(x, y, t),
\end{array}\right.
$$


so that

$$
\left\{\begin{array}{l}
\frac{\sqrt{H}}{H-U^{2}}\left(\partial_{t}+V \partial_{y}\right) h^{*}=\partial_{t_{1}} h_{1}^{*} \\
\left(\partial_{x}-\frac{U}{H-U^{2}}\left(\partial_{t}+V \partial_{y}\right)\right) h^{*}=\partial_{x_{1}} h_{1}^{*} \\
\partial_{y} h^{*}=\sqrt{H-U^{2}} \partial_{y_{1}} h_{1}^{*} \\
\partial_{x}^{P M L} h^{*}=\partial_{x_{1}}^{P M L} h_{1}^{*}+\frac{U}{\sqrt{H}} \partial_{t_{1}} h_{1}^{*} \\
L h^{*}=\left(\left(H-U^{2}\right)\left[\partial_{t_{1}}^{2}-\partial_{x_{1}}^{2}-\partial_{y_{1}}^{2}\right]+f^{2}\right) h_{1}^{*}=L_{1} h_{1}^{*} \\
L^{P M L} h^{*}=\left(\left(H-U^{2}\right)\left[\partial_{t_{1}}^{2}-\partial_{x_{1}}^{P M L^{2}}-\partial_{y_{1}}^{2}\right]+f^{2}\right) h_{1}^{*}=L_{1}^{P M L} h_{1}^{*}
\end{array}\right.
$$

with

$$
\partial_{x_{1}}^{P M L} h_{1}^{*}=\alpha_{1}\left(x_{1}\right) \partial_{x_{1}} h_{1}^{*} \text { and } \alpha_{1}\left(x_{1}\right)=\frac{\partial_{t_{1}}}{\partial_{t_{1}}+\sigma\left(x_{1}\right)} .
$$

Remark 3.3. The operator $\alpha_{1}$ corresponds to the classical PML operator.

With this change of variable the problem becomes

$$
L_{1} h_{1}^{*}=0 \text { for } x_{1}<0 \text { and } L_{1}^{P M L} h_{1}^{*}=0 \text { for } x_{1}>0,
$$

with the transmission conditions

$$
\left[h_{1}^{*}\right]=0 \text { and }\left[D_{x_{1}} h_{1}\right]=0 \quad \text { at } x_{1}=0,
$$

where $D_{x_{1}}$ is defined as $\partial_{x_{1}}$ for $x_{1}<0$ and $\partial_{x_{1}}^{P M L}$ for $x_{1}>0$.

This change of variable is close to the one used by Nataf [1] for the linearized Euler equation. It can also be compared to the ones presented in [10, 14, 11] for the linearized Euler equation in the case of an horizontal mean flow. It is devoted to stabilize the PML by turning the so-called "back-propagative modes" (i.e. the modes whose group velocity is opposed to their phase velocity) which cause the instabilities (see [15]) into regular modes.

Next we use the formal change of variable

$$
\left\{\begin{array}{l}
t_{2}=t_{1}, \\
x_{2}=x_{1}+\frac{1}{\partial_{t}} \int_{0}^{x_{1}} \sigma(x) d x, \\
y_{2}=y_{1}, \\
h_{2}^{*}\left(x_{2}, y_{2}, t_{2}\right)=h_{1}^{*}\left(x_{1}, y_{1}, t_{1}\right),
\end{array}\right.
$$


so that

$$
\left\{\begin{array}{l}
\partial_{t_{1}} h_{1}^{*}=\partial_{t_{2}} h_{2}^{*}, \\
\partial_{x_{1}} h_{1}^{*}=\partial_{x_{2}} h_{2}^{*}, \text { for } x_{2}<0 \\
\partial_{x_{1}} h_{1}^{*}=\left(1+\frac{\sigma\left(x_{2}\right)}{\partial_{t_{2}}}\right) \partial_{x_{2}} h_{2}^{*}=\frac{1}{\alpha_{1}\left(x_{2}\right)} \partial_{x_{2}} h_{2}^{*}, \text { for } x>0 \\
\partial_{y_{1}} h_{1}^{*}=\partial_{y_{2}} h_{2}^{*}, \\
\partial_{x_{1}}^{P M L} h_{1}^{*}=\partial_{x_{2}} h_{2}^{*}, \\
L_{1} h_{1}^{*}=\left(\left(H-U^{2}\right)\left[\partial_{t_{2}}^{2}-\partial_{x_{2}}^{2}-\partial_{y_{2}}^{2}\right]+f^{2}\right) h_{2}^{*}=L_{2} h_{2}^{*}, \\
L_{1}^{P M L} h_{1}^{*}=L_{2} h_{2}^{*},
\end{array}\right.
$$

and the problem becomes

$$
L_{2} h_{2}^{*}=0 \text { for } x_{2}<0 \text { and } L_{2} h_{2}^{*}=0 \text { for } x_{2}>0,
$$

with the transmission conditions

$$
\left[h_{2}^{*}\right]=0 \text { and }\left[\partial_{x_{2}} h_{2}^{*}\right]=0 \quad \text { at } x_{2}=0 .
$$

This change of variable is actually the inverse of the classical one which turns $\partial_{x_{2}}$ into $\partial_{x_{1}}^{P M L}$ used to construct a PML.

We then get that $h_{2}^{*}$ satisfies the Klein-Gordon equation both in the physical and the PML media, with perfect transmission conditions, so that there is no reflected waves in this problem. From the expression of the two changes of variable we used, it can be easily verified that this implies that no waves is reflected neither in the original problem, which completes the proof of the theorem.

\section{Stability of the new PML}

In this section, we assess the attenuation capability of the absorbing layer and for that purpose, we apply a modal analysis. A straightforward study consists in searching for $h^{*}$ under the form

$$
h^{*}(x, y, t)=\left\{\begin{array}{ll}
e^{\lambda x+i(\omega t+k y)} & \text { for } x<0 \\
e^{\lambda^{\mathrm{PML}} x+i(\omega t+k y)} & \text { for } x>0
\end{array},\right.
$$

solution to the problem

$$
\begin{cases}L h^{*}+f^{2} h^{*}=0, & \text { for } x<0, \\ L^{\mathrm{PML}} h^{*}+f^{2} h^{*}=0, & \text { for } x>0, \\ {\left[h^{*}\right]=0 \text { and }\left[D_{x} h^{*}\right]=0 \text { at } x=0,} & \end{cases}
$$


for any $(\omega, k) \in \mathbb{R}^{2}$. Into the absorbing layer, the attenuation factor is related to the $\Re e\left(\lambda^{\mathrm{PML}}\right)$ whose sign must be negative to strongly ensure absorption. The exponential decreasing of the solution into the layer is very interesting from a numerical point of view since the layer can be cut by a boundary on which simple boundary conditions can be used such as Dirichlet or Neumann conditions. Since these conditions are perfectly reflecting, they are not convenient to define a bounded computational domain except if the solution is strongly attenuated into the layer. Indeed the reflections are then very small amplitude-valued and the numerical solution is not impacted into the layer, if it must be extended.

Theorem 4.1. Modal solutions are exponentially decreasing in the $x$-direction, for any $\omega$ and $k$

Proof 4.2. To simplify the analysis, we apply successively the two changes of variable $h_{1}^{*}\left(x_{1}, y_{1}, t_{1}\right)=h^{*}(x, y, t)$ and $h_{2}^{*}\left(x_{2}, y_{2}, t_{2}\right)=h_{1}^{*}\left(x_{1}, y_{1}, t_{1}\right)$. Recalling that $h_{2}^{*}$ is solution to the classical transmission problem:

$$
\begin{cases}L_{2} h_{2}^{*}+f^{2} h_{2}^{*}=0, & \text { for } x_{2}<0, \\ L_{2} h_{2}^{*}+f^{2} h_{2}^{*}=0, & \text { for } x_{2}>0, \\ {\left[h_{2}^{*}\right]=0 \text { and }\left[\partial_{x_{2}} h_{2}^{*}\right]=0 \text { at } x_{2}=0,} & \end{cases}
$$

we seek modal solutions which are given by:

$$
h_{2}\left(x_{2}, y_{2}, t_{2}\right)=e^{\lambda_{2} x_{2}+i\left(\omega_{2} t_{2}+k_{2} y_{2}\right)} .
$$

The change of variable implies that:

$$
\left\{\begin{array}{l}
\omega_{2}=\frac{\sqrt{H}}{H-U^{2}}(\omega+V k) \\
k_{2}=\frac{1}{\sqrt{H-U^{2}}} k,
\end{array}\right.
$$

We can then notice that $(\omega, k) \in \mathbb{R}^{2}$ is equivalent to $\left(\omega_{2}, k_{2}\right) \in \mathbb{R}^{2}$ which implies that we can assume now that $\omega_{2}$ and $k_{2}$ are real. By plugging the modal solution into the system, one obtains that there are two solutions $\lambda_{2}^{+}$and $\lambda_{2}^{-}$such that

$$
\begin{cases}\lambda_{2}^{ \pm}= \pm \sqrt{k_{2}^{2}-\omega_{2}^{2}+\frac{f^{2}}{H-U^{2}}}, & \text { if } k_{2}^{2}-\omega_{2}^{2}+\frac{f^{2}}{H-U^{2}} \geq 0, \\ \lambda_{2}^{ \pm}= \pm i \sqrt{\omega_{2}^{2}-k_{2}^{2}-\frac{f^{2}}{H-U^{2}}}, & \text { if } k_{2}^{2}-\omega_{2}^{2}+\frac{f^{2}}{H-U^{2}}<0\end{cases}
$$

In the first case where $k_{2}^{2}-\omega_{2}^{2}+\frac{f^{2}}{H-U^{2}} \geq 0$, the corresponding modes are real and not propagative. Only one of them is physically admissible in each halfspace. For $x_{2}>0$ it is $\lambda_{2}^{-}$while for $x_{2}<0$, it is $\lambda_{2}^{+}$. In the second case where $k_{2}^{2}-\omega_{2}^{2}+\frac{f^{2}}{H-U^{2}}<0$, the modes are imaginary and propagative. When $\omega_{2}>0$, 
$\lambda_{2}^{-}$propagates along the $x$-direction in the positive sense while $\lambda_{2}^{+}$propagates in the opposite sense. Obviously the propagation senses commute when $\omega_{2}<0$.

Now to assess the attenuation factor of the absorbing layer, we study the real part of the corresponding PML mode. We have:

$$
\lambda^{P M L}=\left(1-\frac{i \sigma\left(x_{2}\right)}{\omega_{2}}\right) \lambda_{2}^{ \pm}+i \frac{U}{\sqrt{H}} \omega_{2}
$$

We first consider the mode $\lambda^{P M L}$ corresponding to the mode $\lambda_{2}^{-}$admissible into $\left\{x_{2}>0\right\}$. Then, when $k_{2}^{2}-\omega_{2}^{2}+\frac{f^{2}}{H-U^{2}} \geq 0$, we get:

$$
\Re e\left(\lambda^{P M L}\right)=-\sqrt{k_{2}^{2}-\omega_{2}^{2}+\frac{f^{2}}{H-U^{2}}}<0
$$

Next, if $k_{2}^{2}-\omega_{2}^{2}+\frac{f^{2}}{H-U^{2}}<0$ and $\omega_{2}>0$, only $\lambda_{2}^{-}$induces a wave into the absorbing layer and

$$
\Re e\left(\lambda^{P M L}(x)\right)=-\frac{\sigma\left(x_{2}\right)}{\omega_{2}} \sqrt{\omega_{2}^{2}-k_{2}^{2}-\frac{f^{2}}{H-U^{2}}}<0,
$$

while if $\omega_{2}<0$, the PML mode is generated by $\lambda_{2}^{+}$and we have:

$$
\Re e\left(\lambda^{P M L}\right)=\frac{\sigma\left(x_{2}\right)}{\omega_{2}} \sqrt{\omega_{2}^{2}-k_{2}^{2}-\frac{f^{2}}{H-U^{2}}}<0,
$$

As a conclusion, $\Re e\left(\lambda^{P M L}\right)$ is negative in each case which implies the modal solution is exponentially decreasing into the layer.

Remark 4.3. The modes $\lambda_{2}^{ \pm}$do not depend on $x_{2}$ whereas the corresponding PML modes depend on $x$. This is due to the fact that the symbol of the differential operator $L_{2}+f^{2}$ is independent of $x_{2}$.

If we apply a Dirichlet or a Neumann condition at the end of the PML, we can prove by using the image principle, at it is done for instance in [16], that the reflected waves are also exponentially decreasing.

\section{Discretization of the equation and numerical results}

\subsection{The PML system written as a first order system}

Systems (7) and (10) both involve a second order operator ( $L$ and $\left.L^{\mathrm{PML}}\right)$, whose numerical solution leads to the inversion of a full matrix. To avoid this difficulty, we have chosen, following Nataf [1], to rewrite these systems as systems requiring only the inversion of first order operators. System (7) can obviously be rewritten as $(2)$ and, by using $L^{\mathrm{PML}}=L^{\mathrm{PML}}-L+L$ in the first equation of (10), we obtain:

$$
\left(L^{\mathrm{PML}}-L\right) h^{*}+\left(G^{2}+f^{2}-H \Delta\right) h^{*}=0 .
$$


Next, multiplying respectively by $G \partial_{x}$ and $G \partial_{y}$ the second and the third equation of (10) and adding the resulting equations, we get

$$
\Delta h^{*}=f\left(G \partial_{x} v-G \partial_{y} u\right)-G^{2}\left(\partial_{x} u+\partial_{y} v\right) .
$$

Let us then multiply respectively by $\partial_{y}$ and $\partial_{x}$ the second and the third equation of (10) to obtain:

$$
\left(G \partial_{x} v-G \partial_{y} u\right)=-f\left(\partial_{x} u+\partial_{y} v\right),
$$

so that

$$
\Delta h^{*}=-\left(f^{2}+G^{2}\right)\left(\partial_{x} u+\partial_{y} v\right),
$$

which, plugged in (16), leads to:

$$
\left(G^{2}+f^{2}\right)^{-1}\left(L^{\mathrm{PML}}-L\right) h^{*}+h^{*}-H \partial_{x} u-H \partial_{y} v=0 .
$$

Let us remark that, when $f=0$, we do not exactly recover the PML model proposed by Nataf in [1], this is due to the operator $\left(G^{2}+f^{2}\right)^{-1}$ which, when $f=0$, is nothing but $G^{-2}$, so that the term

$$
G^{-2}\left(L^{\mathrm{PML}}-L\right) h^{*}=G^{-2}\left(L^{\mathrm{PML}}-L\right) G h
$$

can be rewritten as $G^{-1}\left(L^{\mathrm{PML}}-L\right) h$.

For the inversion of the operator $G^{2}+f^{2}$, we introduce two auxiliary variables, $\xi_{1}$ and $\xi_{2}$, such that

$$
\left\{\begin{array}{l}
G \xi_{2}=\left(L^{\mathrm{PML}}-L\right) h^{*}-f^{2} \xi_{1} ; \\
G \xi_{1}=\xi_{2}
\end{array}\right.
$$

and the resulting system is rewritten as

$$
\left\{\begin{array}{l}
h^{*}+\xi_{1}-H \partial_{x} u-H \partial_{y} v=0 \\
G h=h^{*} \\
G u+\partial_{x} h-f v=0 \\
G v+\partial_{y} h+f u=0 \\
G \xi_{2}=\left(L^{\mathrm{PML}}-L\right) h^{*}-f^{2} \xi_{1} \\
G \xi_{1}=\xi_{2}
\end{array}\right.
$$

It should be pointed out that $\xi_{1}$ and $\xi_{2}$ have to be computed in the whole domain, because of the transport operator $G$. Therefore the complete set of equations has to be written as

$$
x<0\left\{\begin{array}{l}
h^{*}-H \partial_{x} u-H \partial_{y} v=0 ; \\
G h=h^{*} ; \\
G u+\partial_{x} h-f v=0 ; \\
G v+\partial_{y} h+f u=0 ; \\
G \xi_{2}=-f^{2} \xi_{1} ; \\
G \xi_{1}=\xi_{2} ;
\end{array} \quad x>0\left\{\begin{array}{l}
h^{*}+\xi_{1}-H \partial_{x} u-H \partial_{y} v=0 \\
G h=h^{*} \\
G u+\partial_{x} h-f v=0 \\
G v+\partial_{y} h+f u=0 \\
G \xi_{2}=\left(L^{\mathrm{PML}}-L\right) h^{*}-f^{2} \xi_{1} \\
G \xi_{1}=\xi_{2}
\end{array}\right.\right.
$$


with the two additional transmission conditions $\left[\xi_{1}\right]=0$ and $\left[\xi_{2}\right]=0$ at $x=0$.

Let us now focus on the operator

$$
L^{P M L}-L=\left(H-U^{2}\right)\left(\partial_{x}^{2}-\left(\partial_{x}^{P M L}\right)^{2}\right)-2 U \partial_{t}\left(\partial_{x}-\partial_{x}^{P M L}\right) .
$$

Since the operator $\partial_{x}^{P M L}$ is a pseudo-differential operator, its numerical discretization is not straightforward and we have to introduce auxiliary functions. This could be done directly for the operator $\partial_{x}^{P M L}$ but we found more convenient to consider $\partial_{x}-\partial_{x}^{P M L}$ since

$$
\partial_{x}^{2}-\left(\partial_{x}^{P M L}\right)^{2}=\partial_{x}\left(\partial_{x}-\partial_{x}^{P M L}\right)+\left(\partial_{x}-\partial_{x}^{P M L}\right) \partial_{x}-\left(\partial_{x}^{P M L}-\partial_{x}\right)^{2} .
$$

Eqs. (8) and (9) yields:

$$
\partial_{x}^{\mathrm{PML}}=\alpha(x)\left(\partial_{x}-\frac{U}{H-U^{2}}\left(\partial_{t}+V \partial_{y}\right)\right)+\frac{U}{H-U^{2}}\left(\partial_{t}+V \partial_{y}\right),
$$

Hence, the operator $\partial_{x}-\partial_{x}^{P M L}$ can be formally written as

$$
\partial_{x}-\partial_{x}^{P M L}=\sigma(x) \frac{\left(H-U^{2}\right) \partial_{x}-\sqrt{H}\left(\partial_{t}+V \partial_{y}\right)}{\left(H-U^{2}\right) \sigma(x)+\sqrt{H}\left(\partial_{t}+V \partial_{y}\right)}
$$

so that the equation $\phi=\left(\partial_{x}-\partial_{x}^{P M L}\right) \psi$ is simply solved using the following system:

$$
\left\{\begin{array}{l}
\left(\sqrt{H}\left(\partial_{t}+V \partial_{y}\right)+\left(H-U^{2}\right) \sigma(x)\right) \phi=\sigma(x)\left(\left(H-U^{2}\right) \partial_{x}-\sqrt{H}\left(\partial_{t}+V \partial_{y}\right)\right) \psi \\
\partial_{t} \psi(t=0)=0
\end{array}\right.
$$

and the equation $\phi=\left(L^{\mathrm{PML}}-L\right) \psi$ is solved by:

$$
\left\{\begin{aligned}
\phi & =\left(H-U^{2}\right)\left(\partial_{x} \xi_{3}+\xi_{4}\right)+2 U \partial_{t} \xi_{3} \\
\xi_{3} & =\left(\partial_{x}-\partial_{x}^{P M L}\right) \psi \\
\xi_{4} & =\left(\partial_{x}-\partial_{x}^{P M L}\right)\left(\partial_{x} \psi+\xi_{3}\right)
\end{aligned}\right.
$$

In summary, the system to be solved in the horizontal layer is

$$
x>0\left\{\begin{array}{l}
h^{*}+\xi_{1}-H \partial_{x} u-H \partial_{y} v=0 \\
G h=h^{*} \\
G u+\partial_{x} h-f v=0 \\
G v+\partial_{y} h+f u=0 \\
G \xi_{2}=\left(H-U^{2}\right)\left(\partial_{x} \xi_{3}+\xi_{4}\right)+2 U \partial_{t} \xi_{3}-f^{2} \xi_{1} \\
G \xi_{1}=\xi_{2} \\
\xi_{3}=\left(\partial_{x}-\partial_{x}^{P M L}\right) h^{*} \\
\xi_{4}=\left(\partial_{x}-\partial_{x}^{P M L}\right)\left(\partial_{x} h^{*}+\xi_{3}\right) .
\end{array}\right.
$$


with the additional conditions at $\xi_{3}=\xi_{4}=0$ at $x=0$.

The procedure for obtaining a vertical PML is very similar to the one we have presented for an horizontal layer. The corner PMLs can be then obtained by applying the PML procedure either to the vertical or to the horizontal layer and we have chosen the second possibility. It is worth noting that the two ways does not lead to same equations in the corner, therefore we can not guarantee that the corner layers are perfectly matched with the vertical layers. However, in all the experiments we have made, we did not notice any reflections at the interface between these layers.

\subsection{Discretization of the equations}

Systems (18) and (2) are solved using a finite difference scheme based on a regular staggered grid of space step $\Delta x=\Delta y$ with a time step $\Delta t$. The unknowns $h, h^{*}, \xi_{1}$ and $\xi_{2}$ and $\xi_{4}$ are computed on each point $\left(x_{i}, y_{j}\right)=(i \Delta x, j \Delta x)$ of the grid . $u$ and $\xi_{3}$ (resp. $\left.v\right)$ are computed at points $\left(x_{i+1 / 2}, y_{j}\right)=((i+$ $1 / 2) \Delta x, j \Delta x)\left(\operatorname{resp} .\left(x_{i}, y_{j+1 / 2}\right)=(i \Delta x,(j+1 / 2) \Delta x)\right)$. All these unknowns are computed at times $t_{n}=n \Delta t$. The operators $G$ and $\partial_{t}+V \partial_{y}$ are discretized by a classical first-order upwind scheme (we suppose here that both $U$ and $V$ are positive), for instance we have:

$$
(G h)_{i, j}^{n}=\frac{h_{i, j}^{n+1}-h_{i, j}^{n}}{\Delta t}+U \frac{h_{i, j}^{n}-h_{i-1, j}^{n}}{\Delta x}+V \frac{h_{i, j}^{n}-h_{i, j-1}^{n}}{\Delta y} .
$$

and

$$
\left(\partial_{t} \xi_{3}+V \partial_{y} \xi_{3}\right)_{i+1 / 2, j}^{n}=\frac{\xi_{3}^{n+1}{ }_{i+1 / 2, j}^{n+1}-\xi_{3 i+1 / 2, j}^{n}}{\Delta t}+V \frac{\xi_{3 i+1 / 2, j}^{n}-\xi_{3 i+1 / 2, j-1}^{n}}{\Delta x}
$$

The other space derivatives are discretized with second order centered schemes, for instance

$$
\left(\partial_{x} u\right)_{i, j}^{n}=\frac{u_{i+1 / 2, j}^{n}-u_{i-1 / 2, j}^{n}}{\Delta x}
$$

The CFL condition of the scheme is $\Delta t \leq 0.3 \frac{\Delta x}{\sqrt{H}}$, these value has been computed experimentaly.

\subsection{Numerical results}

In the following experiments we have considered a computational domain $D=[0 ; 10 H] \times[0 ; 10 H]$, and a PML of width $\delta=2.5 H$, inside away from the boundary of $D$ in section 2 . At the ends of the layers, we have to impose a transparent condition for the vorticity waves. Let us recall that these waves satisfy $\partial_{x} u+\partial_{y} v=0$, or, from the first equation of (2), $G h=0$, which is a classical transport equation. Moreover, the condition $G h=0$ on the outflow boundary can be easily imposed thanks to (22). At the inflow boundary, the condition $h=0$ should be sufficient, nevertheless, the discretization of the equations creates an artificial vorticity mode, which has the same speed as the physical one, but in the opposite direction. This mode has a very small amplitude compared 
to the physical mode, but its reflection at the inflow boundary can produce numerical instabilities and we have chosen to absorb it by imposing an "inverse transport condition" (i.e. $\left(\partial_{t}-U \partial_{x}-V \partial_{y}\right) h=0$ ) instead of $h=0$ at the inflow boundary. In all experiments, we have used an absorption profile $\sigma=\sigma_{m}\left(\frac{d}{\delta}\right)^{\beta}$, with $\beta=2$. The damping $\sigma_{m}$ has been chosen empirically equal to 20 . To validate our method, we have compared our results to the ones obtained on a larger computational domain $D_{r e f}=[0 ; 50 H] \times[0 ; 50 H]$. We have calculated the relative error in time for an oblique uniform mean flow near the upper corner (two points inside the free region away from the interface). Full initial conditions for the shallow water equations are tested with radial Gaussian pulses as follows (cf. [9]):

$$
\begin{aligned}
& h(x, y, 0)=A e^{\frac{\left(x-x_{0}\right)^{2}+\left(y-y_{0}\right)^{2}}{R^{2}}} \\
& u(x, y, 0)=2 \sqrt{H} \frac{y-y_{0}}{R^{2}} A e^{\frac{\left(x-x_{0}\right)^{2}+\left(y-y_{0}\right)^{2}}{R^{2}}} \\
& v(x, y, 0)=-2 \sqrt{H} \frac{x-x_{0}}{R^{2}} A e^{\frac{\left(x-x_{0}\right)^{2}+\left(y-y_{0}\right)^{2}}{R^{2}}}
\end{aligned}
$$

where $A$ denotes the amplitude, $\left(x_{0}, y_{0}\right)$ is the center of the pulse and $R$ its radius. We have set $A=0.1,\left(x_{0}, y_{0}\right)=(5 H, 5 H)$, and $R=H / 6$. We have tested four values: 1, 10, 100 and 1000 for the characteristic depth $H$. We consider an oblique mean flow such that $U=V \geq 0$ (orientation of 45 degrees) and for each value of $H$, we have tested two values of the Froude number $F=$ $\sqrt{U^{2}+V^{2}} / \sqrt{H}=0.2$ and 0.6 and we have set $f=1 / \sqrt{H}$. With this choice of parameter, the Rossby radius $r_{E}=\sqrt{H} / f$ is simply equal to $H$. The space step is $\Delta x=0.05 H$ and the time step is chosen accordingly to the above CFL condition. The results are reported in figures 1-11. We can observe that the maximum error is obtained when long waves are predominant which corresponds to low Rossby radius $r_{E}=1$. For the other values of $r_{E}$, the dynamic is dominated by short waves and the results are better. Let us however remark that, whatever the value of $r_{E}$ is, the relative errors are always below $0.02 \%$.

We also have tested the model for parallel mean flow and we have obtained satisfactory results as in $[10,9]$.

Since the non rotating shallow water system is self contained in the new PML model, we have tested our PML in configuration used by Nataf [1] by setting $f=0, H=1$ and $F=0.2$ (see figures 14 and 15). As we said before, the two PML are different because of the inversion of the operator $G^{2}+f^{2}$, and our PML may not have been well suited to such problems. However, we obtained a relative error less than $0.5 \%$ with our system, which indicates that the PML does produce too much spurious reflexion even when $f=0$.

To study the long time stability of the method, we have reproduced each of the above experiments during time intervals 1000 times larger (which represents about $10^{6}$ time steps). No instability was observed for various cases, which numerically proves the long-time stability of the method. The theoretical study 
of the stability is a work in progress and will be the object of a forthcoming paper.
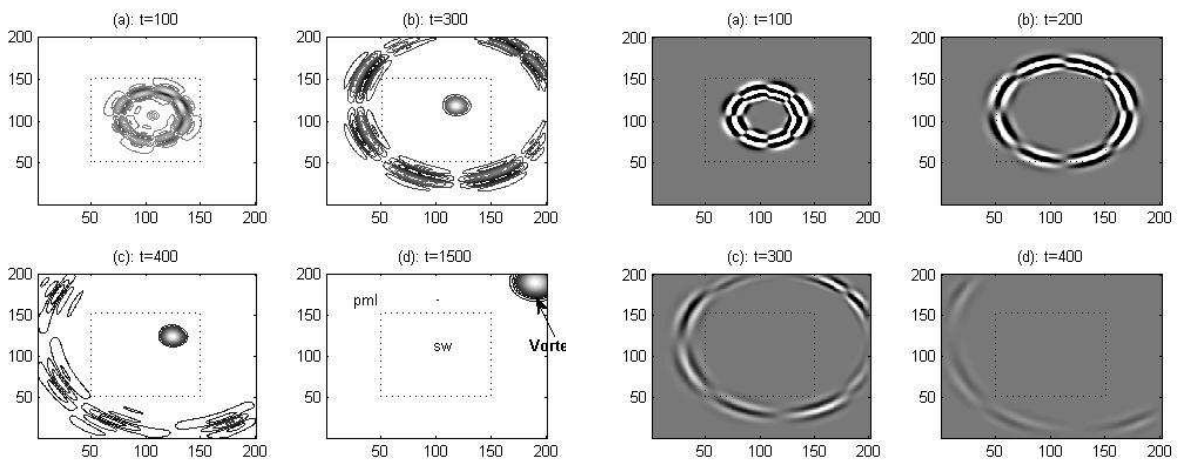

Figure 1: Height potential contours at Rossby radius $r_{E}=1000$ and Froude number $F_{r}=0.2$ flow.

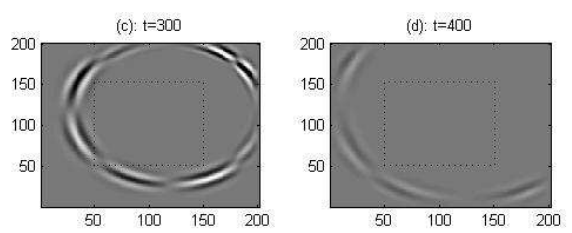

Figure 2: Advective component of the height potential contours at Rossby radius $r_{E}=$ 1000 and Froude number $F=0.2$ flow.

\section{Conclusion}

We have constructed a new absorbing layer which is perfectly matched to the linearized shallow water system. The construction process is applied to a second-order formulation of the equations which allows one to uncouple the height of water from the velocity field. By this way, only waves that can produce reflections at the interface between the physical domain and the absorbing one are damped. Then we have rewritten the PML equations as a first-order system by introducing auxiliary unknowns and numerical investigations have been developed on the first-order formulation. The numerical results allowed us to assess the performance of the PML condition which is efficient and seems to be stable for long time simulation. The analysis of stability of the new PML system is a current work and we aim at proving that the PML condition does not produce long time unstabilities.

\section{Acknowledgements}

These results were partially obtained during M. Tlemcani was visiting the team-project Magique-3d. The authors acknowledge the support by Egide Program. Any opinions, findings, conclusions or recommendations expressed in this material are those of the authors and do not necessarily reflect the views of INRIA. 

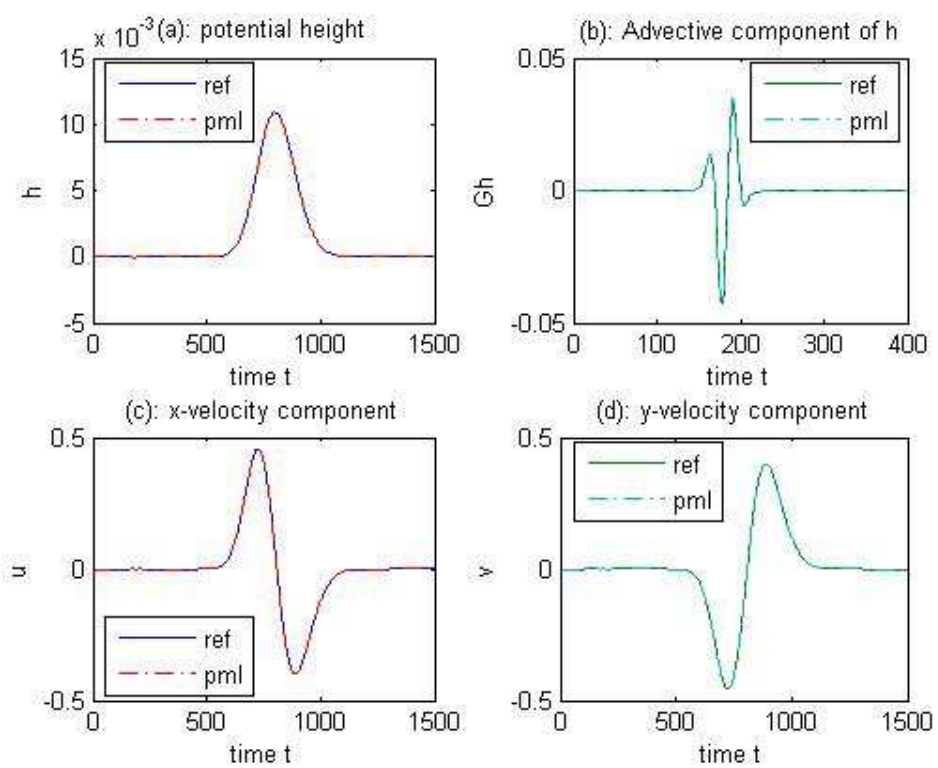

Figure 3: Reference and pml solutions vs. time near the upper corner at Rossby radius $r_{E}=1000$ and Froude number $F=0.2$ flow.

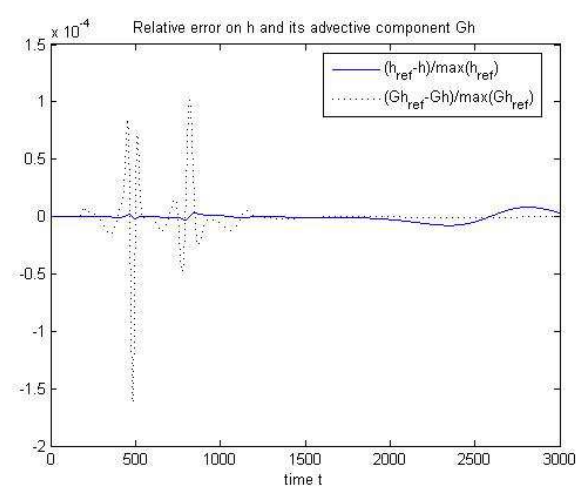

Figure 4: Relative error on $h$ and $G h$ vs. time at Rossby radius $r_{E}=1000$ and Froude number $F=0.2$ flow (the vortex passes without absorption).

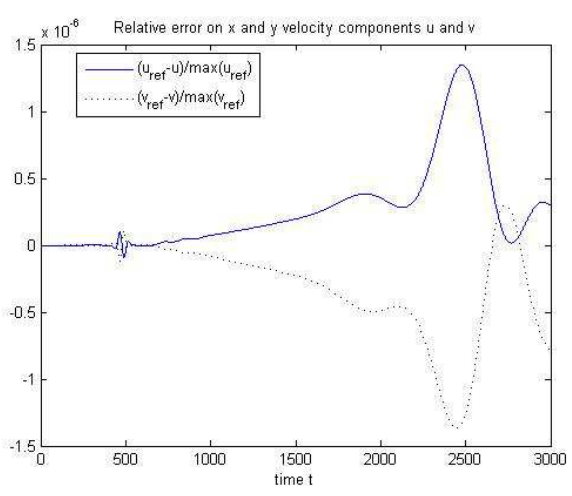

Figure 5: Relative error on $u$ and $v$ vs. time at Rossby radius $r_{E}=1000$ and Froude number $F=0.2$ flow. 
(a): $t=100$

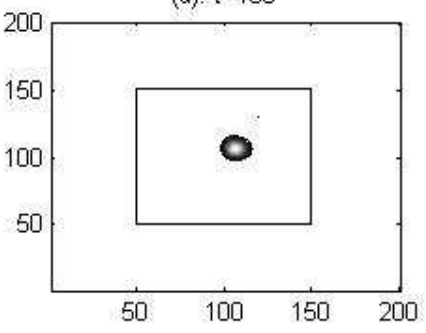

(c): $t=800$

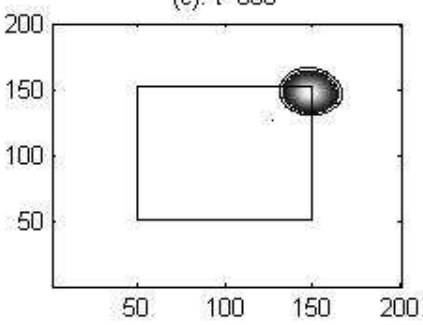

(b): $t=600$

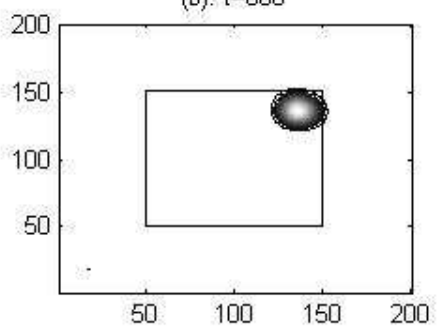

(d): $t=1500$

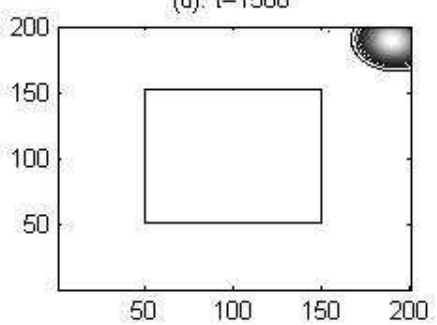

Figure 6: Height potential contours at Rossby radius $r_{E}=1$ and Froude number $F=0.2$ flow.

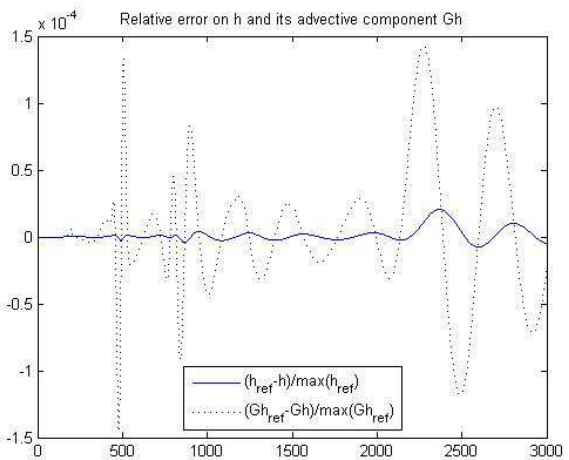

Figure 7: Relative error on $h$ and $G h$ vs. time at Rossby radius $r_{E}=1$ and Froude number $F=0.2$ flow.

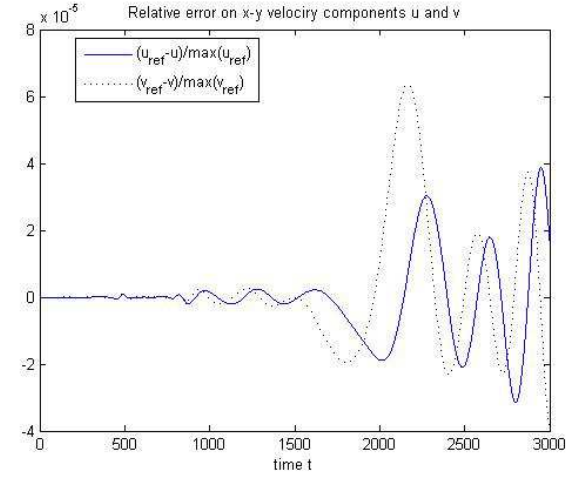

Figure 8: Relative error on $u$ and $v$ vs. time at Rossby radius $r_{E}=1$ and Froude number $F_{r}=0.2$ flow. 
(a): $t=100$

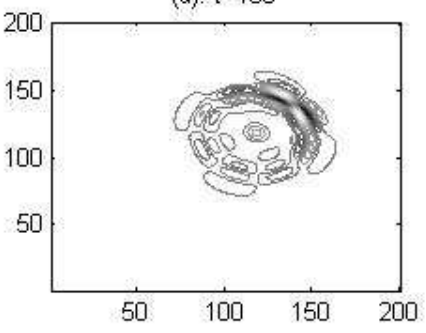

(c): $t=400$

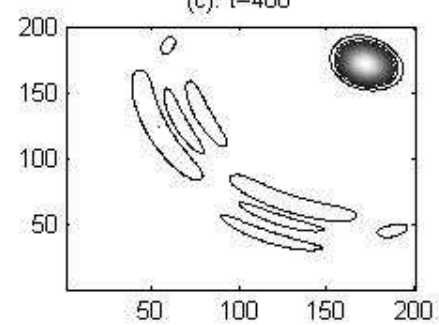

(b): $\mathrm{t}=300$

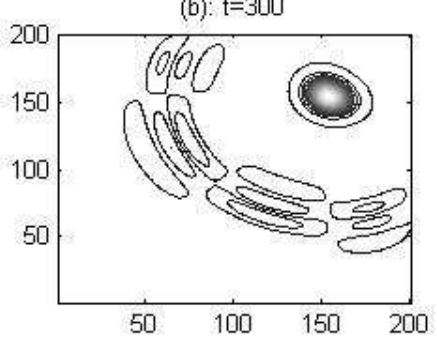

(d): $\mathrm{t}=600$

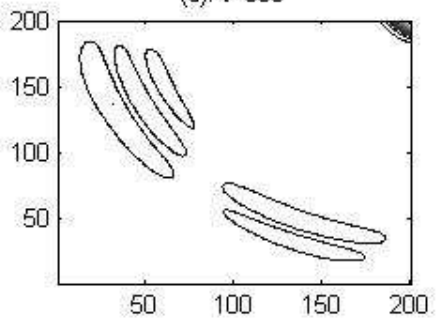

Figure 9: Height potential contours at Rossby radius $r_{E}=1000$ and Froude number $F_{r}=0.6$ flow.

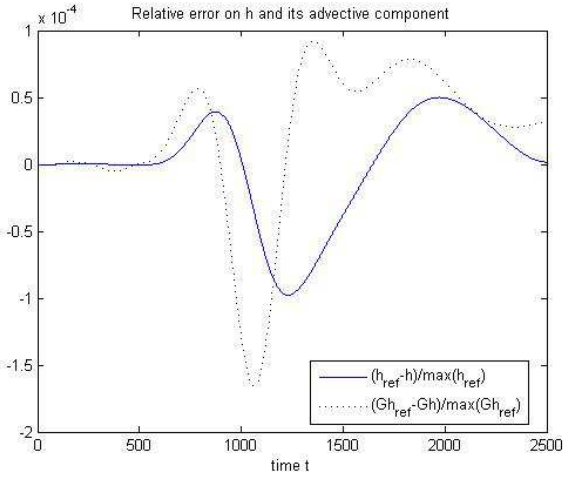

Figure 10: Relative error on $h$ and $G h$ vs. time at Rossby radius $r_{E}=1000$ and Froude number $F=0.6$ flow.

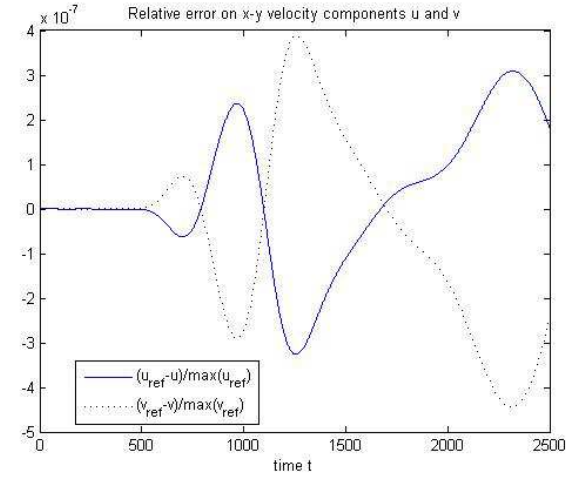

Figure 11: Relative error on $u$ and $v$ vs. time at Rossby radius $r_{E}=1000$ and Froude number $F_{r}=0.6$ flow. 


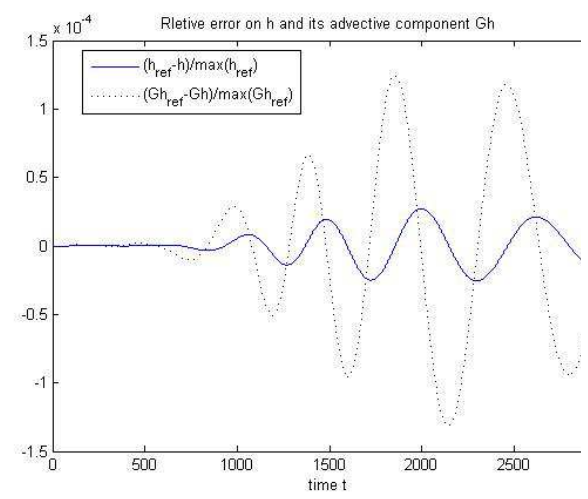

Figure 12: Relative error on $h$ and $G h$ vs. time at Rossby radius $r_{E}=1$ and Froude number $F=0.6$ flow.

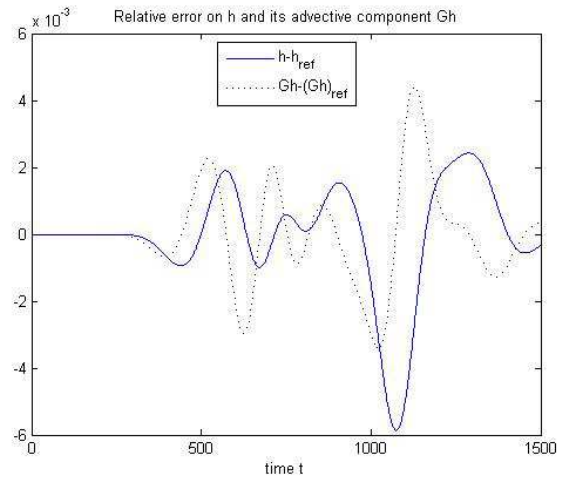

Figure 14: Relative error on $h$ and $G h$ vs. time for the non rotating sw-equations at $f=$ 0 , and Froude number $F=0.2$ flow

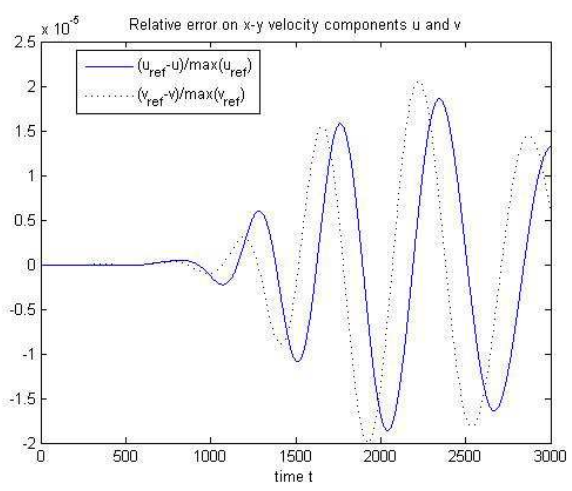

Figure 13: Relative error on $u$ and $v$ vs. time at Rossby radius $r_{E}=1$ and Froude number $F=0.6$ flow.

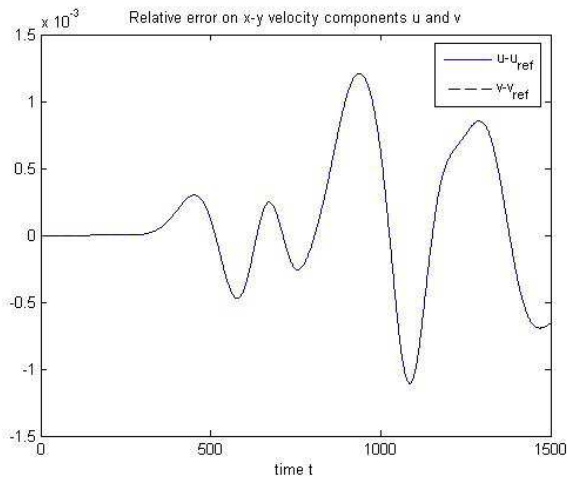

Figure 15: Relative error on $u$ and $v$ vs. time for the non rotating sw-equations at $f=0$, and Froude number $F=0.2$ flow. 


\section{References}

[1] F. Nataf, A new approach to perfectly matched layers for the linearized euler equations, J. Comput. Phys. 214 (2) (2006) 757-772.

[2] M. Gunzburger, G. Guirguis, Error estimates and implementation issues for artificial boundary conditions methodes in exterior problems, in: Advances in computer methods for partial differential equations, Vol. VI, IMACS, 1983, pp. pp. 338-345.

[3] S. Tsynkov, Numerical solution of problems in unbounded domains, a review, Appl. Numer. Math. 27 (1998) 465-532.

[4] J. Pedlosky, Geophysical Fluid Dynamics, Springer, New York, 1987.

[5] V. Joolen, B. Neta, D. Givoli, A stratified dispersive wave model with highorder nonreflecting boundary conditions, Computers and Mathematics with Applications 8 (2008) 1167-1180.

[6] R. Higdon, adiation boundary conditions for dispersive waves, SIAM J. Numer. Anal. 31.

[7] J. Bérenger, A perfectly latched layer for the absorption of electromagnetic waves, J. Comput. Phys. 114 (2) (1994) 185-200.

[8] J. Bérenger, Three-dimensional perfectly matched layer for the absorption of electromagnetic waves, J. Comput. Phys. 127 (2) (1996) 363-379.

[9] I. Navon, B. Neta, M. Hussaini, A perfectly matched layer approach for the linearized shallow water equations models, Monthly Weather Review 132 (2004) 1369-1378.

[10] F. Hu, A perfectly matched layer absorbing boundary condition for linearized euler equation with a non-uniform flow, J. of Comp. Phys. 208 (2) (2005) 469-492.

[11] T. Hagstrom, A new construction of perfectly matched layers for hyperbolic systems with applications to the linearized euler equation, in: Mathematical and Numerical aspects of Wave propagation, WAVES 2003, Springer, Berlin, 2003, pp. 125-129.

[12] I. Lie, Well-posed transparent boundary conditions for the shallow water equations, Applied Numerical Mathematics 38 (2001) 445-474.

[13] J. Wloka, B. Rowley, B. Lawruk, Boundary Value Problems for Elliptic Systems, cambridge Edition, Cambridge University Press, 1995.

[14] S. Abarbanel, D. Gottlieb, J. S. Hesthaven, Well-posed perfectly matched layers for advective acoustics, J. Comput. Phys. 154 (2) (1999) 266-283. 
[15] E. Bécache, S. Fauqueux, P. Joly, Stability of perfectly matched layers, group velocities and anisotropic waves, J. Comput. Phys. 188 (2) (2003) 399-433.

[16] J. Diaz, P. Joly, A time domain analysis of pml models in acoustics, Comput. Methods Appl. Mech. Engrg. 195 (29-32) (2006) 3820-3853. 